\title{
Luminosity and mass functions of galactic open clusters
}

\section{NGC $4852^{\star, \star \star}$}

\author{
G. Carraro ${ }^{1,2,3}$, G. Baume ${ }^{1,4}$, G. Piotto ${ }^{1}$, R. A. Méndez ${ }^{2,5}$, and L. Schmidtobreik ${ }^{5}$
}

1 Dipartimento di Astronomia, Università di Padova, Vicolo dell’Osservatorio 5, 35122 Padova, Italy

2 Departamento de Astronomía, Universidad de Chile, Casilla 36-D, Santiago, Chile e-mail: gcarraro@das.uchile.cl

3 Astronomy Department, Yale University, PO Box 208101, New Haven, CT 06520-8101, USA

${ }^{4}$ Facultad de Ciencias Astronómicas y Geofísicas de la UNLP, IALP-CONICET, Paseo del Bosque s/n, La Plata, Argentina

5 ESO-Chile, Casilla 19001, Santiago, Chile

Received 23 April 2004 / Accepted 8 February 2005

\begin{abstract}
We present wide-field deep UBVI photometry for the previously unstudied open cluster NGC 4852 down to a limiting magnitude $I \sim 24$, obtained from observations taken with the Wide Field Imager camera on-board the MPG/ESO $2.2 \mathrm{~m}$ telescope at La Silla (ESO, Chile). These data are used to obtain the first estimate of the cluster basic parameters, to study the cluster spatial extension by means of star counts, and to derive the Luminosity (LF) and Mass Function (MF). The cluster radius turns out to be $5.0 \pm 1.0$ arcmin. The cluster emerges clearly from the field down to $V=20$ mag. At fainter magnitudes, it is completely confused with the general Galactic disk field. The stars inside this region define a young open cluster (200 million years old) $1.1 \mathrm{kpc}$ far from the Sun $(m-M=11.60, E(B-V)=0.45)$. The Present Day Mass Functions (PDMF) from the $V$ photometry is one of the most extended in mass obtained to date, and can be represented as a power-law with a slope $\alpha=2.3 \pm 0.3$ and (the Salpeter MF in this notation has a slope $\alpha=2.35$ ), in the mass range $3.2 \leq \frac{m}{m_{\circ}} \leq 0.6$.

Below this mass, the MF cannot be considered as representative of the cluster MF, as the cluster merges with the field and therefore the MF is the result of the combined effect of strong irregularities in the stellar background and interaction of the cluster with the dense Galactic field. The cluster total mass at the limiting magnitude results to be $2570 \pm 210 M_{\odot}$.
\end{abstract}

Key words. open clusters and associations: individual: NGC 4852 - Hertzsprung-Russell (HR) and C-M diagrams

\section{Introduction}

This paper is the second in a series (Prisinzano et al. 2001, hereafter Paper I) devoted to the study of the stellar Luminosity (LF) and Mass Functions (MF) in Galactic Open Clusters as a function of age and position in the Galactic disk. The goal is to probe the environmental dependence of the Initial Mass Function (IMF) in open star clusters.

Unfortunately, this is a challenging task for several reasons. First, most open clusters are poorly populated ensembles of stars, containing from tens to hundreds of stars, and only in rare cases up to some thousands. Secondly, they are located in the inner regions of the thin disk. This fact makes it very difficult to segregate cluster members (as opposed to globular clusters), due to the high contamination from field stars located in

^ Based on observations carried out at the European Southern Observatory, La Silla, Chile.

$\star \star$ Table 3 is only available in electronic form at the CDS via anonymous ftp to cdsarc.u-strasbg.fr $(130.79 .128 .5)$ or via http://cdsweb.u-strasbg.fr/cgi-bin/qcat?J/A+A/436/527 the line of sight of the clusters. Third, they can be strongly obscured due to interstellar absorption between us and the cluster.

Therefore, to obtain cluster membership is a cumbersome process. This is feasible for clusters close to the Sun, for which we can obtain high quality radial velocity and proper motion measurements. Farther away one has to rely on statistical corrections which are usually made by comparing the cluster with the Galactic disk field in its outskirts (see Paper I, Kalirai et al. 2003; and Burke et al. 2004, for similar studies).

Ideally, when a correction for field star contamination has been performed, a reasonable Present Day Mass Function (PDMF) can be derived from the observed LF. By modeling the dynamical evolution of the cluster the PDMF can be converted into an IMF which can be compared with the MFs of other clusters to look for universality or deviations.

In Paper I we discussed the intermediate-age open cluster NGC $4815\left(l=303^{\circ} .63, b=-2.09\right)$, for which we found that the PDMF presents a slope $\alpha \approx 3$ in the mass range $2.5 \leq \frac{m}{m} \leq 0.8$. In this paper we present results for NGC 4852 (VDBH $143, l=304^{\circ} .03, b=3.25$ ), a seemingly younger open 

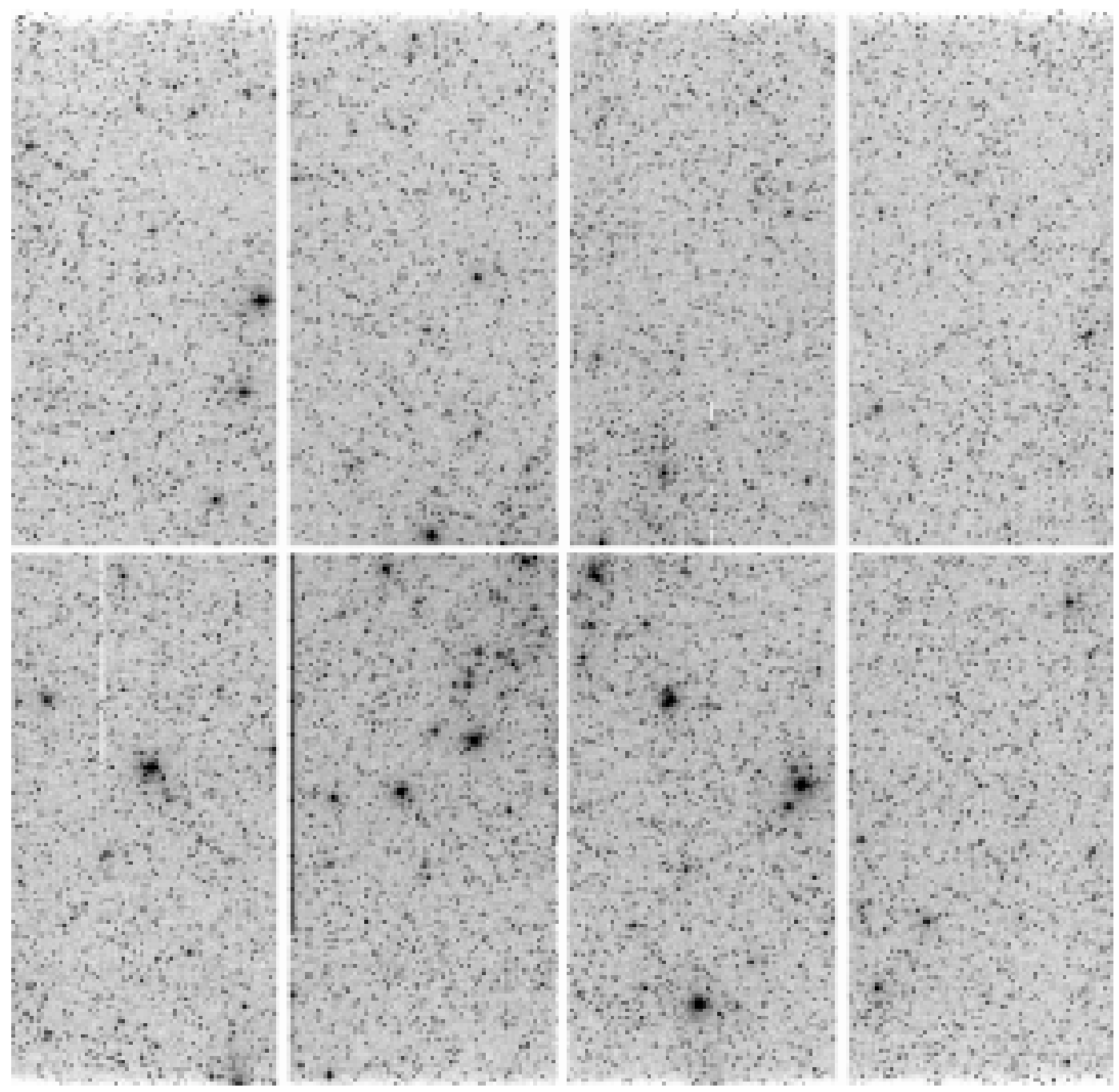

Fig. 1. A deep WFI image of the region around NGC 4852 taken in $V$ filter. The field center is RA $=13^{\mathrm{h}} 00^{\mathrm{m}} 06^{\mathrm{s}}$ and $\mathrm{Dec}=-59^{\circ} 36^{\prime} 02^{\prime \prime}(\mathrm{J} 2000)$. Each chip covers an area of $8.12 \times 16^{\prime} .25$, while the full field of view is of $34^{\prime} \times 33^{\prime}$. The cluster center is located not far from the center of the image, and the cluster has an apparent radius of about $2-3$ arcmin.

cluster never studied before, observed with the WFI camera attached to the $2.2 \mathrm{~m}$ telescope in La Silla.

The size of the eight CCD mosaic allows us to obtain a good representation of the field stellar population in the direction of the cluster to be used for a statistical subtraction of foreground/background objects.

The layout of the paper is as follows. In Sect. 2 we present the observations and data reduction technique; Sect. 3 presents a detailed analysis of the star counts and a determination of the cluster size, while Sect. 4 is dedicated to a discussion of the CMDs. Section 5 illustrates the derivation of the cluster's basic parameters. Section 6 deals with the LF, while Sect. 7 presents the MF of NGC 4852 and the derivation of the cluster mass. Finally, Sect. 8 summarizes our results.

\section{Observations and reduction}

\subsection{Observations}

The open cluster NGC 4852 has been observed using the Wide Field Imager (WFI) camera mounted at the Cassegrain focus of
Table 1. Log of the observations of the open cluster NGC 4852, July 7 , 2002. The field was centered at RA $=13: 00: 06$, Dec $=-59: 36: 02$.

\begin{tabular}{crcc}
\hline \hline Field & Airmass & Filter & Exp. times [s] \\
\hline NGC 4852 & 1.239 & $V$ & $1,30,900$ \\
& 1.294 & $I$ & $1,30,600$ \\
& 1.196 & $B$ & $1,60,1200$ \\
& 1.169 & $U$ & $1,30,600$ \\
\hline
\end{tabular}

the MPG/ESO $2.2 \mathrm{~m}$ Telescope at La Silla (Chile). This camera is a $4 \times 2$ mosaic of $2 \mathrm{~K} \times 4 \mathrm{~K} \mathrm{CCD}$ detectors (see Fig. 1 ). The scale is $0{ }^{\prime} 238 /$ pix, therefore the mosaic covers $34^{\prime} \times 33^{\prime}$ and, due to the narrow inter-chips gaps, the filling factor is $95.9 \%$. Data were obtained on the photometric night of July 7, 2002 with seeing values near to $1^{\prime \prime}$. Details of the observed fields and exposure times are listed in Table 1. 
Table 2. Coefficients of the calibration equations.

\begin{tabular}{ccc}
\hline \hline$u_{1}=-2.87 \pm 0.01$ & $u_{2}=0.46$ & $u_{3}=-0.05 \pm 0.02$ \\
$b_{1}=0.36 \pm 0.02$ & $b_{2}=0.27$ & $b_{3}=-0.26 \pm 0.03$ \\
$v_{1}=0.91 \pm 0.02$ & $v_{2}=0.12$ & $v_{3}=+0.06 \pm 0.02$ \\
$i_{1}=2.00 \pm 0.03$ & $i_{2}=0.06$ & $i_{3}=-0.16 \pm 0.02$ \\
\hline
\end{tabular}

\subsection{Data reduction and calibration}

All the images were pre-processed in a standard way with the IRAF $^{1}$ package CCDRED and using the sets of bias and sky flat-field images collected during the same observing night. Instrumental magnitudes and positions of the stars for each frame were derived by profile-fitting photometry with the DAOPHOT package, using the Point Spread Function (PSF) method (Stetson 1987). In order to obtain the transformation equations relating the instrumental $(u, b, v, i)$ magnitudes to the standard $U B V$ (Johnson), I (Kron-Cousins) system, we followed the procedure already described in Baume et al. (2004). Four Landolt (1992) fields of standards have been observed. Specifically: SA-092 (23 stars), SA-104 (20 stars), SA-107 (19 stars) and SA 110 (23 stars). For all of these stars, aperture photometry was obtained on all the images. The transformation coefficients and the final calibrated photometry were computed by using the package PHOTCAL. We used transformation equations of the form:

$u=U+u_{1}+u_{2} * X+u_{3}(U-B)$

$b=B+b_{1}+b_{2} * X+b_{3}(B-V)$

$v=V+v_{1}+v_{2} * X+v_{3}(V-I)$

$i=I+i_{1}+i_{2} * X+i_{3}(V-I)$

where the values of the coefficients are listed in Table 2. In these equations $u b v i$ are the aperture magnitudes already normalized to $1 \mathrm{~s}$, and $X$ is the airmass. Second-order color terms were tried and turned out to be negligible in comparison to their uncertainties. Unfortunately, a few bright stars were saturated in $B$ and $U$.

In Fig. 2 we present the photometric errors trends as a function of the magnitude $V$. The error in $V$ is below 0.1 mag up to $V=20$. The global $\mathrm{rms}$ of the calibration is $0.02 \mathrm{mag}$ both for all the filters. Our photometry consists of 145925 stars (Table 3 ) and will be made available electronically at the CDS.

\subsection{Astrometry}

The astrometric solution is a basic step to combine the eight chips and to put all the stars in the same coordinate system. In order to obtain an astrometric solution we use the SkyCat tool and the Guide Star Catalogue v2 (GSC-2) at ESO. This way we find about 1500 stars in each chip for which we have both the celestial coordinates on the GSC-2 and the corresponding pixel coordinates. Then, by using the IRAF tasks CCXYMATCH, CCMAP and CCTRAN, we find the corresponding

${ }^{1}$ IRAF is distributed by NOAO, which are operated by AURA under cooperative agreement with the NSF.

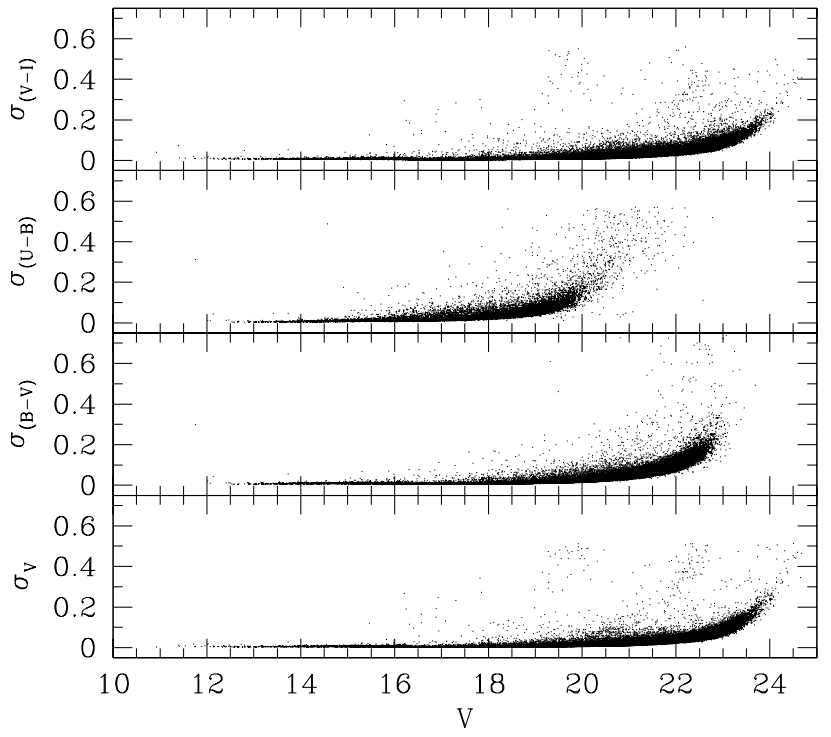

Fig. 2. Trend of the photometric errors in $V,(V-I),(B-V)$ and $(U-B)$ as a function of the magnitude $V$.

transformations between the two coordinate systems for each chip and compute the individual celestial coordinates for all the detected stars. The transformations have an rms value of 0!29, in agreement with other studies (Momany et al. 2001; Prisinzano et al. 2004). The results are displayed in Fig. 3, where all the reference stars are considered.

\subsection{Artificial star tests}

In order to obtain the LF, we estimated the completeness of our sample. Completeness corrections have been determined by standard artificial-star experiments on our data (see Piotto \& Zoccali 1999; and Baume et al. 2004). We selected only the stars having fitting parameters $\chi \leq 1.0$ and $A B S$ (sharp) $\leq 0.2$, and located in the Color Magnitude Diagram (CMD) inside the strip shown in Fig. 8 (dashed lines, see the discussion below).

Only the stars selected this way will be used in all the following analysis. We created 5 artificial images by adding artificial stars to the original images. In order to avoid overcrowding, in each experiment we added at random positions only $15 \%$ of the original number of stars. The artificial stars had the same color and luminosity distribution as the original sample. The incompleteness, defined as the ratio of the found stars over the added artificial stars is listed in Table 4 for $V$ magnitudes. Since the stars in each bin has both $V$ and $I$ magnitudes, we derive the incompleteness level also for the $I$ magnitude by adjusting the $I$ bins according to the star color. Due to the relatively low crowding the magnitude migration effects (Piotto \& Zoccali 1999) were negligible. A good rule of thumb is that LF counts should not be corrected by a factor greater than 2 .

In the LFs we include only the values for which the completeness corrections, defined as the ratio between the number of found artificial stars to that of the original added ones, was $50 \%$ or higher. Moreover we do not consider the bin between 23 and $24 \mathrm{mag}$, since the error is very large. Therefore we set the limiting magnitude to $V=23$. 

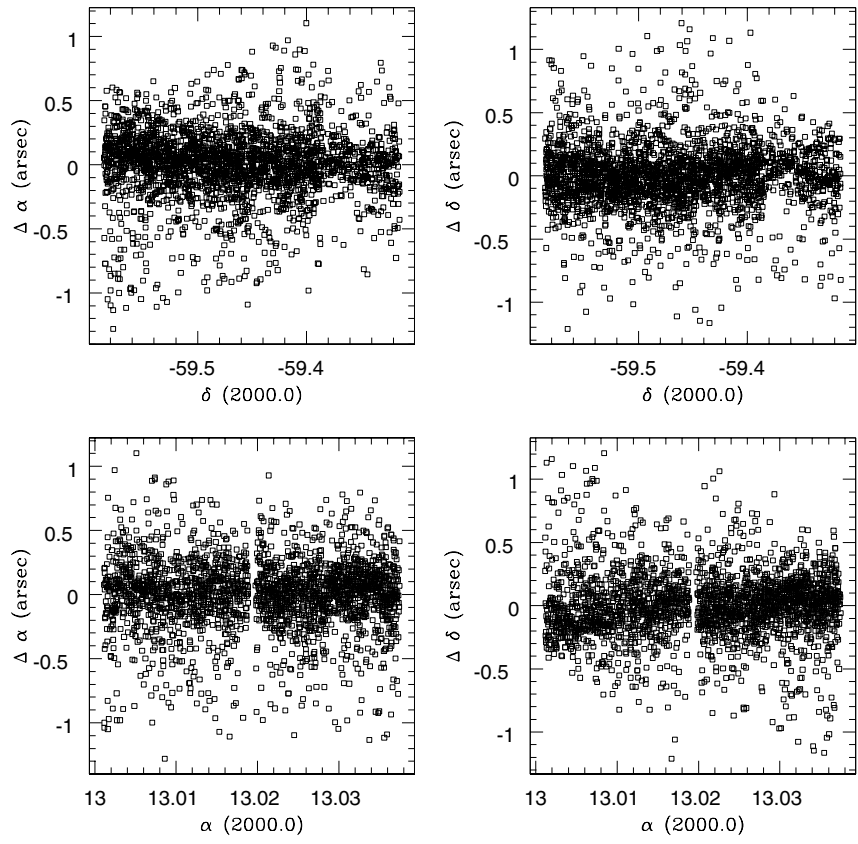

Fig. 3. Positional residuals on the right ascension and declination transformations obtained from a comparison of our astrometric solution and the GSC 2.2 reference catalog as a function of RA and Dec for all the eight CCDs of the WFI mosaic.

Table 4. Completeness analysis results.

\begin{tabular}{cc}
\hline \hline$\Delta V$ & NGC 4852 \\
mag & $\%$ \\
\hline $15-16$ & $99.8 \pm 15.4$ \\
$16-17$ & $97.1 \pm 14.0$ \\
$17-18$ & $97.6 \pm 13.7$ \\
$18-19$ & $97.5 \pm 9.6$ \\
$19-20$ & $93.9 \pm 7.6$ \\
$20-21$ & $86.5 \pm 5.9$ \\
$21-22$ & $88.2 \pm 4.5$ \\
$22-23$ & $88.7 \pm 16.0$ \\
$23-24$ & $69.1 \pm 71.6$ \\
\hline
\end{tabular}

\section{Star counts and cluster size}

The aim of this section is to obtain the surface density distribution of NGC 4852 and to derive the cluster size in the magnitude space by means of star counts. The cluster radius is indeed one of the most important cluster parameters, useful (together with cluster mass) for a determination of cluster dynamical parameters. Star counts allow us to determine statistical properties of clusters (as visible star condensations) with respect to the surrounding stellar background.

NGC 4852 appears as a concentration of bright stars in a region of about 4-5 arcmin. In order to derive the radial stellar surface density we first look for the highest peak in the stellar density to determine the cluster center. The adopted center is placed at $\alpha=13: 00: 06.5 ; \delta=-59: 36: 02.0$, which is very close to that given by Dias et al. (2002). Then, the radial density profile is constructed by performing star counts inside increasing concentric annuli 0.5 wide around the cluster center and then by dividing by their respective surfaces. This is done as

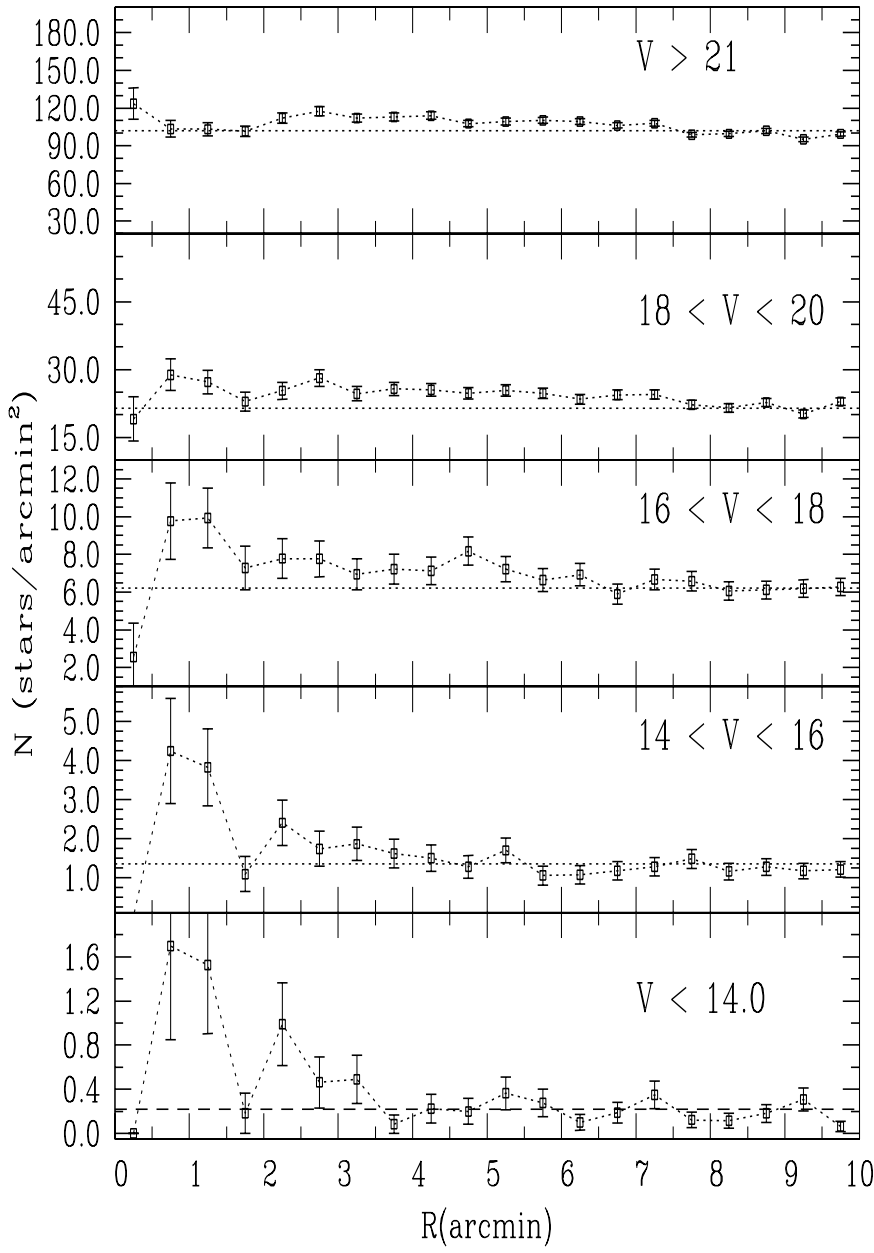

Fig. 4. Star counts as a function of radius from the adopted cluster center for various magnitude intervals. The dashed line in each panel indicates the mean density level of the surrounding Galactic disk field in that magnitude level.

a function of apparent magnitude and compared with the mean density of the surrounding Galactic field in the same brightness interval. The contribution of the field has been estimated through star counts in the region outside 9 arcmin from the cluster center. Poisson standard deviations have also been computed and normalized to the area of each ring as a function of the magnitude, both for the cluster and for the field.

The result is shown in Fig. 4, where one readily sees that NGC 4852 significantly emerges from the mean field above $V \approx 20$. At fainter magnitudes the cluster is confused with the Galactic disk population. Based on the radial density profiles in Fig. 4, we find that stars brighter than $V=16$ provide a cluster radius smaller than 3.0 arcmin, whereas in the interval $16 \leq V \leq 20$ the cluster radius is somewhat larger (around 5.5 arcmin). This situation is compatible with the cluster having experienced some mass segregation, as a consequence of which massive stars sank toward the cluster center, while lower mass stars were spread toward the cluster envelope. Therefore we propose that the cluster possesses a core-corona structure result of dynamical evolution, with most of the bright stars located inside the core ( 3 arcmin in radius). 


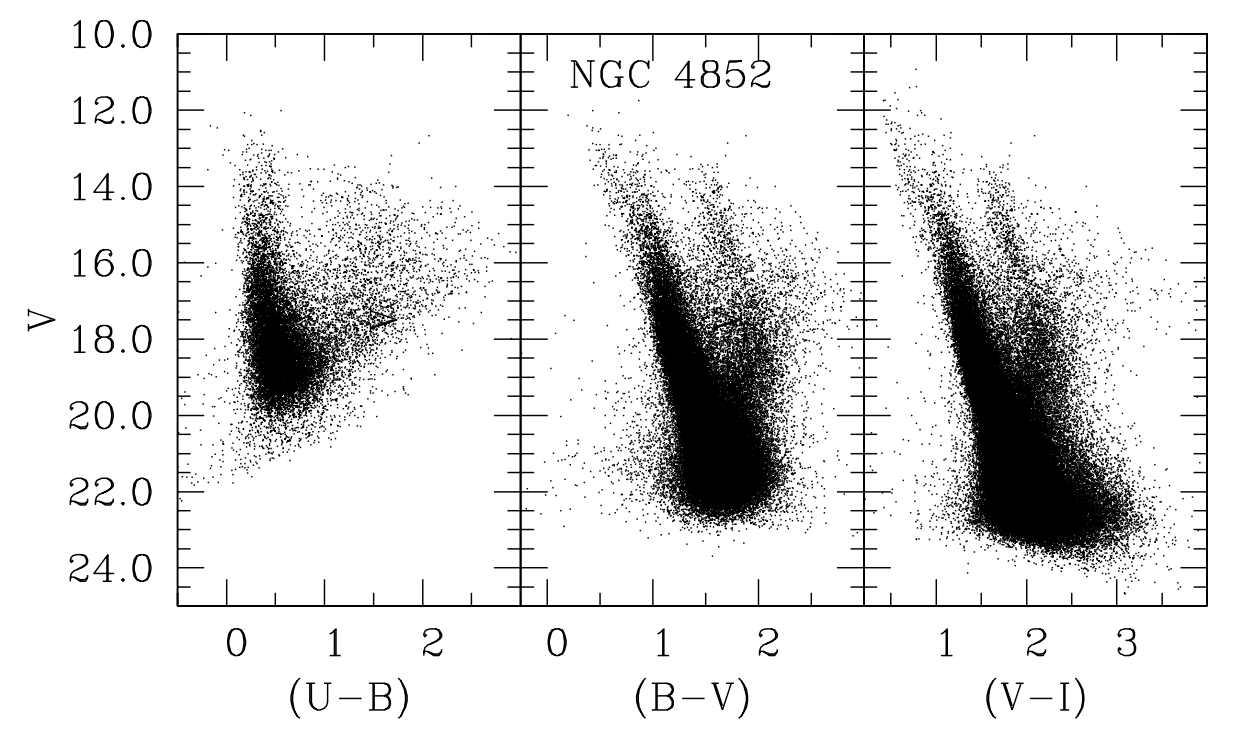

Fig. 5. Mosaic CMDs of NGC 4852.

We adopt as a final estimate of the radius the values $5.0 \pm$ $1.0 \mathrm{arcmin}$. This is larger than the estimate reported by Dias et al. (2002), which was based on a visual inspection. We shall adopt this values of the cluster radius throughout this work.

We stress however that this radius is not the limiting radius of the cluster, but the distance to the cluster center at which the cluster population starts to be confused with the field population.

\section{The color-magnitude diagrams}

We obtained UBVI photometry to determinine the LF (and MF) of the Main Sequence (MS) stars in NGC 4852. We used four colors as we wanted also to derive Two Color Diagrams and the CMDs, which allow us to: i) discriminate between stars and false detections; ii) better identify the cluster population, in particular the MS stars; and iii) derive estimates of the cluster fundamental parameters.

The mosaic CMDs for NGC 4852 are shown in Fig. 5 for the $V$ vs. $(U-B), V$ vs. $(B-V)$ and $V$ vs. $(V-I)$ combination, in the left, middle and right panel, respectively. Our photometry reaches $V=23.5$ at the base of the MS in the $V$ vs. $(V-I)$ diagram (right panel).

The MS extends almost vertically for more about $10 \mathrm{mag}$, from $V=13$ to $V=23.5$, although the completeness analysis prevents us from using stars dimmer than $V=23$. The width of the MS becomes larger at fainter magnitudes. This is partly due to the increasing photometric errors at increasing magnitudes (see Fig. 2). However the MS is much wider than expected simply from photometric errors.

The various causes that contribute to enlarging the natural MS width include the presence of unresolved binary stars (common in open clusters), the contamination by foreground stars, a possible spread in metallicity, and the differential reddening across the cluster area. To have an indication of the importance of differential reddening we used FIRB extinction maps from Schlegel et al. (1998). Across the 30 square arcmin covered by our photometry the typical reddening variations amount to $0.2 \mathrm{mag}(E(B-V)$ in the range $0.70-0.90$ mag, but see also next section).

Therefore we conclude that differential reddening is not the major cause of the MS broadening, but this is probably mainly due to the contamination from foreground field stars.

The CMDs in Fig. 5 show a red sequence which detaches from the MS at $V \approx 19$ (middle and right panels) and which corresponds to the Red Giant Branch of the Galactic disk population. At about $V=14.5$ there seems to be a bifurcation which deserves further attention.

To better clarify this issue, we provide in Fig. 6 a zoom of the upper MS of the CMDs in the right panel of Fig. 5 $(V$ vs. $(V-I))$. In the left panel we show the MS of all the stars located within 3.0 arcmin from the nominal cluster center. In the middle panel we show the stars located at $4.55 \leq r[\operatorname{arcmin}] \leq 5.192$, whereas in the right panel we show the CMD of the field population, sampled outside the cluster radius, at $10.82 \leq r$ arcmin] $\leq 11.105$. The limits have been selected in order to keep the sampling area fixed.

Figure 6 confirms the findings of star counts (see previous section). Most of the cluster bright star $(V \leq 14)$ population is located inside $3.0 \mathrm{arcmin}$ (left panel). This population does not have a counterpart in the field population presented in the right panel. The few bright stars in this CMD are much redder than the mean MS color at the same mag level in the left panel CMD. However a few bright MS stars are still present outside the cluster inner region (middle panel).

On the other hand, stars fainter than $V \approx 16$ are also present outside the cluster central region (middle and right panels), as already emerged from star counts. The group of star in the right panel at $15 \leq V \leq 15.5$ appear redder than the mean MS color at this magnitude and we believe that they are field stars.

The confusion with the stellar field is so high that we cannot conclude anything about the cluster population below $V \approx 17$.

The overall morphology of these CMDs confirm the mass segregation scenario that emerged from star counts and provides an estimate of the radius around 5 arcmin. 


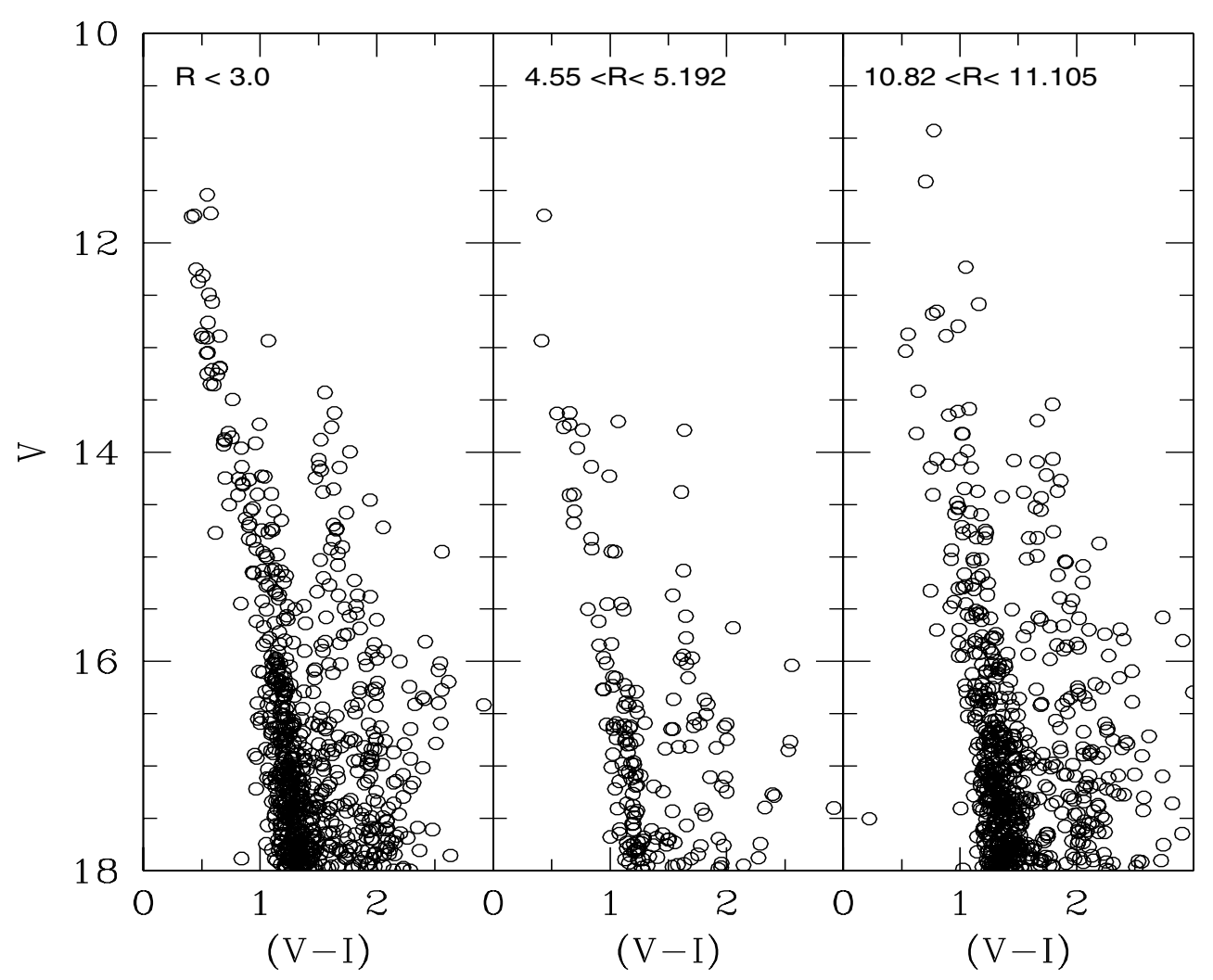

Fig. 6. A zoom of the upper part of the VI CMD for NGC 4852 in different spatial locations. Left panel: stars with 3.0 arcmin. Middle panel: stars at $4.55 \leq r[\operatorname{arcmin}] \leq 5.192$. Right panel: stars at $10.82 \leq r[\operatorname{arcmin}] \leq 11.105$.

Figure 6 suggests that NGC 4852 is indeed an open cluster, presumably young, highly contaminated, that underwent dynamical evolution and mass segregation.

Finally, the bifurcation visible in Fig. 5 can be explained as the contribution of the cluster population, responsible for the blue arm, and the contamination of foreground stars, responsible for the red arm.

\section{Cluster fundamental parameters}

In order to estimate the cluster's fundamental parameters, we consider only the stars located inside 5.0 arcmin and fulfilling the selection criteria defined in Sect. 2. This is because we want to limit the star contamination from the field and because the cluster MS is better defined by the brightest stars.

These stars are shown in Fig. 7. In the left panel we present the fit in the Two Color Diagram, which provides a reddening $E(B-V)=0.45 \pm 0.05$ (error by eye). Unfortunately, a few bright stars were saturated in $B$ and $U$, and therefore the sequence is populated only starting from a spectral type of about B8. Nonetheless, the fit is very good, and allows us also to see that the amount of differential reddening is small, of the order of $0.1 \mathrm{mag}$, as suggested by FIRB maps (see Sect. 4).

We also derived $E(V-I)$ by using the ratio $\frac{E(V-I)}{E(B-V)}=1.244$ from Dean et al. (1978), and it gives $0.56 \pm 0.05 \mathrm{mag}$, and it turns out to match pefectly the ZAMS fitting estimate in the right panel of the same figure. There we show the upper MS in the $V$ vs. $(V-I)$ plane. An empirical ZAMS (dashed line) from Schmidt-Kaler (1982, dashed line) is overimposed shifted by
$(m-M)_{V}=11.60 \pm 0.30 \mathrm{mag}, E(V-I)=0.56 \pm 0.05 \mathrm{mag}$ (errors by eye). The empirical ZAMS well reproduces the distribution of the stars, especially the bulk of the bright stars, which better identify the cluster.

By adopting the normal value for the total to selective absorption $\left(R=\frac{A_{V}}{E(B-V)}=3.1\right)$, we derive an absolute distance modulus $(m-M)_{0}=10.20$, which places the cluster $1.1 \mathrm{kpc}$ from the Sun. Finally, $(m-M)_{I}$ turns out to be $11.0 \mathrm{mag}$.

For the age, the cluster appears young, with all the stars still in the MS. Only the two brightest stars in the CMD, although at different levels, seem to be in the process of leaving the MS. We have tried to superimpose a few solar metallicity isochrones from the Padova models (Girardi et al. 2000), for ages ranging from 10 to $300 \mathrm{Myr}$, and found that the $200 \mathrm{Myr}$ isochrone (solid line in Fig. 7, right panel) accounts very well for the brightest star. Therefore we believe that the cluster cannot be older than this age.

\section{Luminosity function}

The completeness analysis performed in Sect. 2 allows us to derive the LF. This has been constructed as follows:

(i) we selected stars within the adopted cluster radius (see Sect. 3) and according to the fitting parameters (see Sect. 2). Moreover we used only the MS stars, which we looked for by considering the stars located within the two ridge-lines shown in Fig. 8, defined by the two relations $V=6.5(V-I)+17.8$ and $V=6.5(V-I)+12.0$. This is 


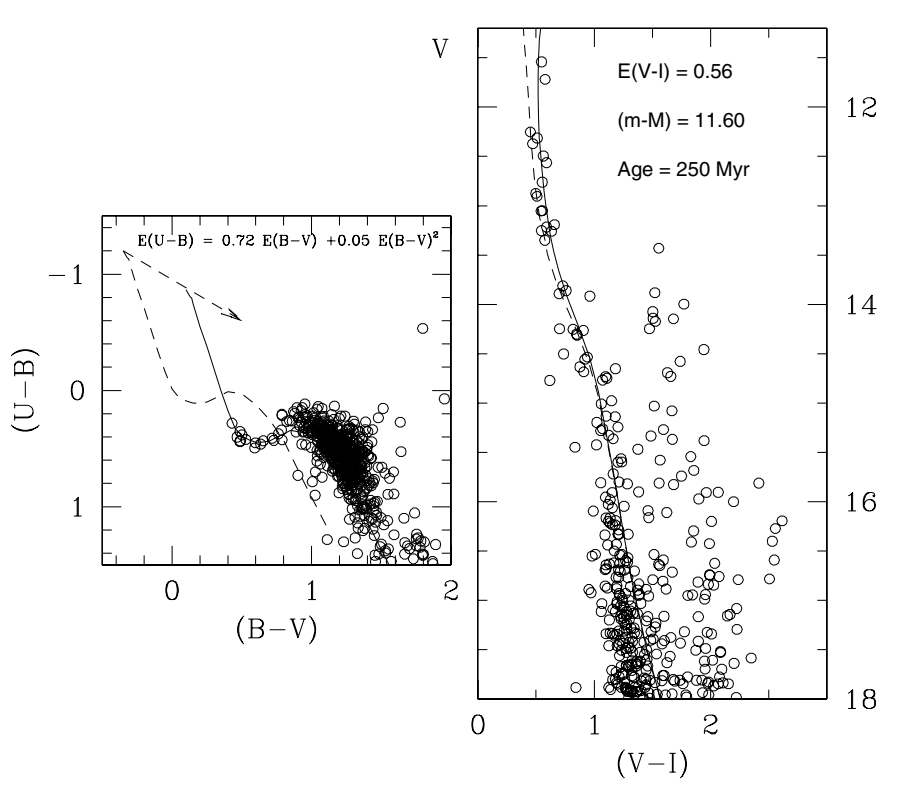

Fig. 7. Left panel: two color diagram for the NGC 4852 (star within the cluster radius). The dashed line is an empirical Schmidt-Kaler (1982) ZAMS, while the solid line is the same ZAMS shifted by $E(B-V)=0.45, E(U-B)=0.72 \times E(B-V)+0.05 \times E(B-V)^{2}$. The dashed arrow indicates the reddening vector. Right panel: the CMD of NGC 4852 (star within the cluster radius). The dashed line is an empirical Schmidt-Kaler (1982) ZAMS shifted by $E(V-I)=0.56$ and $(m-M)=11.60$, while the solid line is a solar metallicity isochrone for the age of $200 \mathrm{Myr}$.

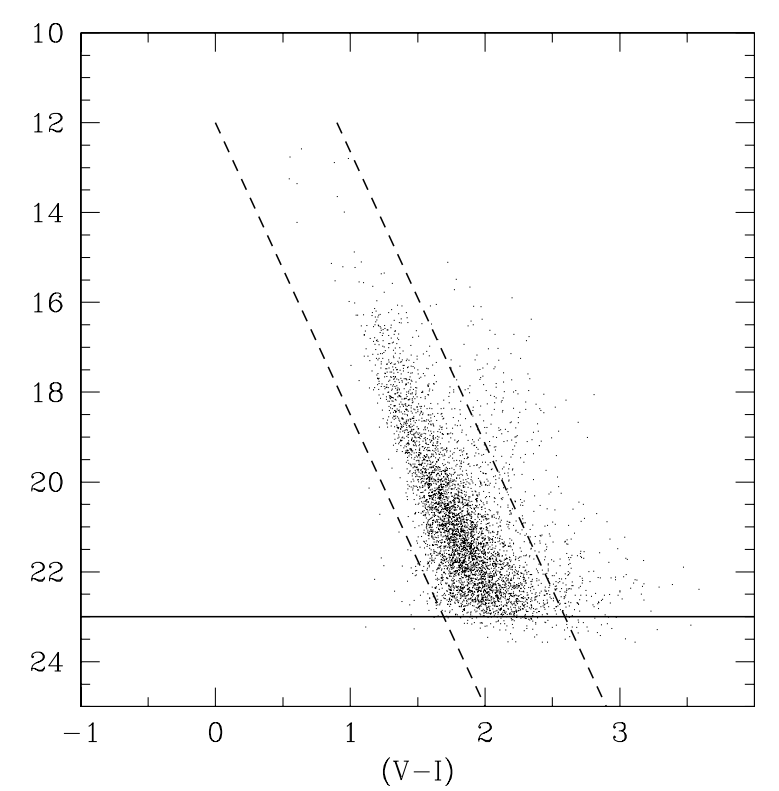

Fig. 8. The CMD of NGC 4852 stars within the adopted cluster radius ( 5 arcmin). The solid horizontal line indicates the completeness limit, whereas the two dashed tilted lines confine the region where we consider MS stars in the cluster are more probably located.

mainly to avoid contamination coming from the Galactic disk RGB population (see also Fig. 5);

(ii) we used the standard histogram technique, and adopted bins $0.5 \mathrm{mag}$ wide. Therefore we counted the number of stars in the cluster area starting from $V=12.0 \mathrm{mag}$. The

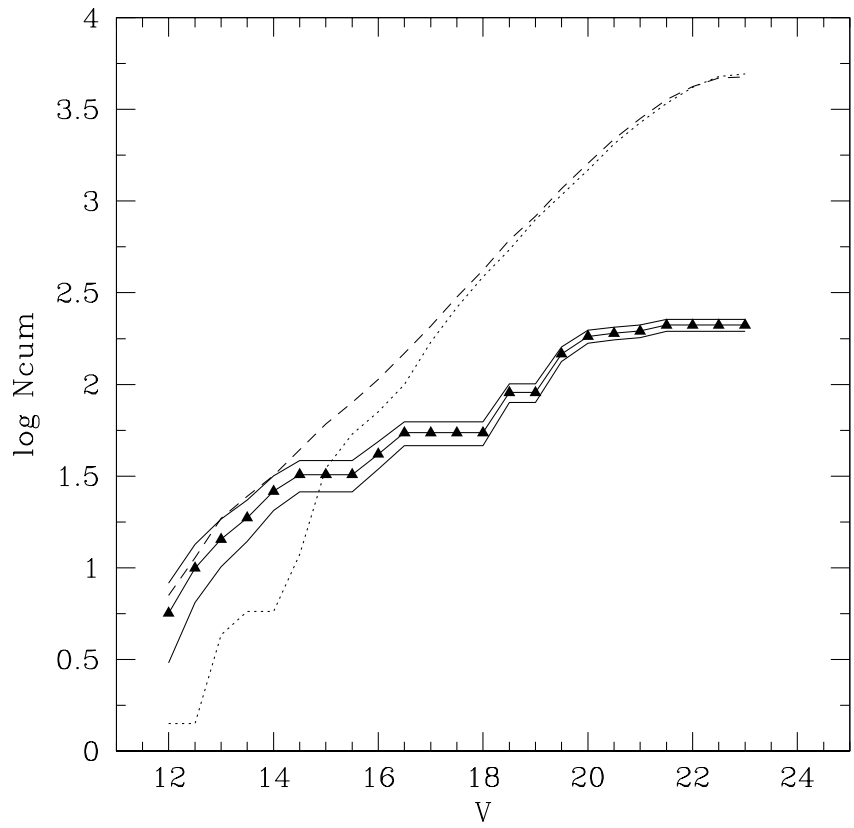

Fig. 9. Luminosity Function (solid triangles) in the $V$ band of NGC 4852 after incompleteness correction and statistical field star subtraction. The contribution of the total sample and the field stars are shown with a dashed and a dotted line.

contribution from the Galactic field was derived outside the cluster region from a corona with the same area of the cluster. For the field we also counted the number of stars in the corona starting from $V=12.0 \mathrm{mag}$.

After having completeness-corrected (see Table 4) both the cluster and the field counts, we derived the NGC 4852 LF by subtracting, bin by bin, the field area counts from the cluster area ones. At this point we computed the logarithm of the cumulative counts distribution, e.g. the logarithm of the fieldcorrected number of stars fainter than a given magnitude.

These are presented in Fig. 9 where we plot the logarithm of the cumulative counts distribution $\left(\log N_{\text {cum }}\right)$ as a function of the magnitude $V$ for the total sample (dashed line), field stars (dotted line) and the LF (filled triangles).

The solid line passing through the filled triangles is the resulting LF, and the two other solid lines below and above are the lower an upper boundaries of the LF due to errors in the LF derivation.

These errors take into account the Poisson errors of the counts both in the cluster and in the field population, and the errors derived from the completeness corrections. We adopted (see Table 4 ) a limiting magnitude $V=23$ as completeness limits. The resulting $\mathrm{LF}$ is a raising function down to $V \approx 20 \mathrm{mag}$. Below that, the contributions of the stars from the cluster and the field are basically the same, and the LF does not increase significantly anymore.

\section{Mass function}

The LFs can be transformed into MFs using a mass-luminosity relation (MLR). Since we could not obtain an empirical transformation, we must rely on theoretical models. Therefore, we 


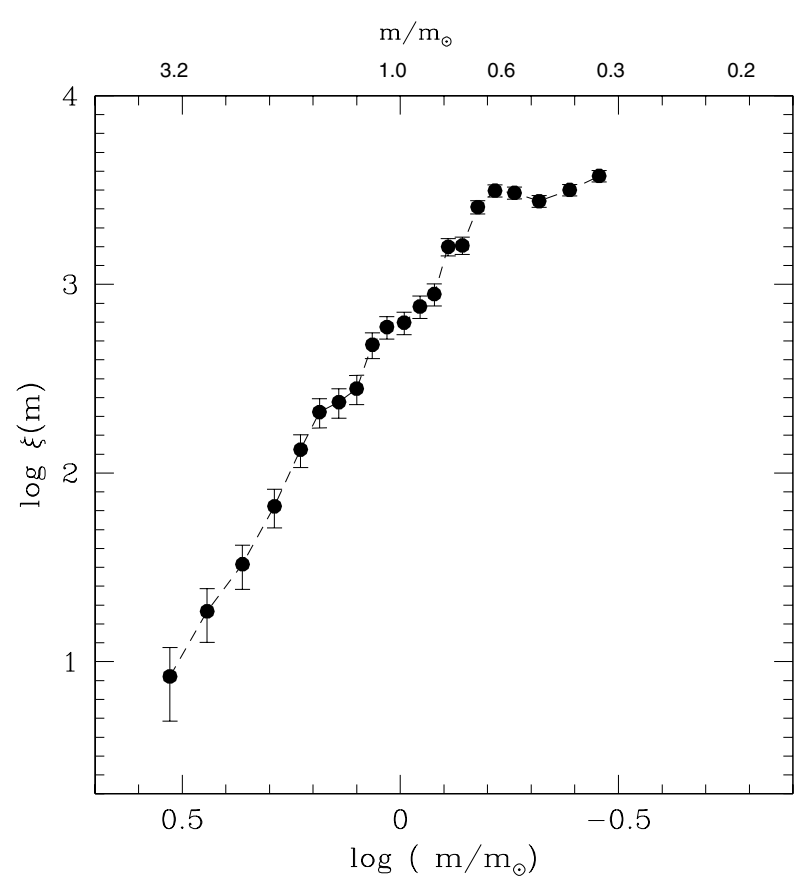

Fig. 10. The MFs of NGC 4852 derived from LFs.

used the ZAMS relation by Girardi et al. (2000) with a metallicity $Z=0.020$. The distance modulus and the reddening are those derived in Sect. 5 .

The resulting MF, obtained from the LF, is shown in Fig. 10. The MF is monotonically increasing up to $\log \left(\frac{m}{m_{\odot}}\right)=-0.2$ (say $M=0.60 M_{\odot}$ ). This corresponds to $V=20.0$, which is roughly the magnitude at which the cluster merges with the field (see Fig. 4). Afterwards, the MF does not increase significantly anymore.

Over the mass range $3.2 \leq \frac{m}{m_{0}} \leq 0.6(12 \leq V \leq 20)$ the MF is a clear power law with a slope $\alpha=2.3 \pm 0.3$, basically consistent with the Salpeter one, which in our notation has a slope of 2.35 .

Finally, it is possible to obtain an estimate of the cluster mass by integrating the luminosity function. In detail,

$$
M=\langle m\rangle \times N=N \int_{M_{V_{\min }}}^{M_{V_{\max }}} m\left(M_{V}\right) \phi\left(M_{V}\right) \mathrm{d} M_{V}
$$

where $M_{V}$ is the absolute stellar magnitude $M_{V}=V-(m-M)_{V}$, $\phi\left(M_{V}\right)$ is the normalized luminosity function

$\phi\left(M_{V}\right)=f\left(M_{V}\right) / N$

$N$ is the total number of stars, $m\left(M_{V}\right)$ is the adopted mass-luminosity relation. The luminosity function and massluminosity relation have been approximated by a spline. The limits in the integral are $M_{V_{\min }}=0.4(V=12)$ and $M_{V_{\max }}=$ $8.4(V=20)$. The integral turns out to be:

$M \simeq 2570 \pm 210 M_{\odot}$.
This mass estimate refers to the limiting magnitude of $V=20.0$, and represents a lower limit of the cluster total mass, due to the effects of field star contamination.

The error is determined from the mass estimate for the upper and lower boundaries of the LF (see Fig. 9).

\section{Discussion and conclusions}

In this paper we have presented the first study of the young open cluster NGC 4852, for which we have obtained wide field deep UBVI CCD photometry. The cluster turns out to be young, with a maximum age of $200 \mathrm{Myr}$, and it is located at $1.1 \mathrm{kpc}$ from the Sun.

We have derived LF and the present-day MF for the cluster. The MF is one of the most extended in mass so far obtained (see Paper I, Fig. 9), and we find that the MF slope is basically consistent with the classical Salpeter one over the whole mass range, without any significant slope change. According to Scalo (1998) the mean slope for the MF in clusters younger than half a Gyr is $\alpha=2.25 \pm 0.88$, and NGC 4852 reflects this behavior. Below $0.6 m_{\odot}$, the MF is no longer reliable, since the cluster is completely confused with the general Galactic disk field.

Acknowledgements. G. Carraro deeply thanks Y. Momany and E. Held for many fruitful discussions and acknowledges financial support from ESO during a visit to Vitacura (Santiago) and from the Fundación Andes. R.A.M. acknowledges support from the Chilean Centro de Astrofísica FONDAP No. 15010003. The work of G. Baume has been supported by Padova University through a postdoctoral grant.

\section{References}

Baume, G., Moitinho, A., Giorgi, E. E., et al. 2004, A\&A, 417, 961

Baume, G., Vázquez, R. A., Carraro, G., et al. 2003, A\&A, 402, 549

Burke, C. J., Gaudi, B. S., \& DePoy, D. L. 2004, AJ, 127, 2382

Dean, J. F., Warren, P. R., \& Cousins, A. W. J. 1978, MNRAS, 183, 569

Dias, W. S., Alessi, B. S., Moitinho, A., et al. 2002, A\&A, 389, 871

Girardi, L., Bressan, A., Bertelli, G., \& Chiosi, C. 2000, A\&AS, 141, 371

Kalirai, J. S., Fahlmam, G. G., Richer, H. B., et al. 2003, AJ, 126, 1402

Landolt, A. U. 1992, AJ, 104, 340

Momany, Y., Vandame, B., Zaggia, S., et al. 2001, A\&A, 379, 452

Piotto, G., \& Zoccali, M. 1999, A\&A, 345, 485

Prisinzano, L., Carraro, G., Piotto, G., et al. 2001, A\&A, 369, 851

Prisinzano, L., Micela, G., Sciortino, S., et al. 2004, A\&A, 417, 945

Salpeter, E. E. 1955, ApJ, 129, 608

Scalo, J. J. 1998, in The Stellar Initial Mass Function, 38th Herstmonceux Conference, ASP. Conf. Ser., 142, 201

Schlegel, D. J., Finkbeiner, D. P., \& Davis, M. 1998, ApJ, 500, 525

Schmidt-Kaler, Th. 1982, Landolt-Börnstein, Numerical data and Functional Relationships in Science and Technology, New Series, Group VI, Vol. 2(b), ed. K. Schaifers, \& H. H. Voigt (Berlin: Springer Verlag), 14

Stetson, P. B. 1987, PASP, 99, 191 